\title{
Gene expression profiling of monocytes displaying herpes simplex virus 1 induced dysregulation of antifungal defences
}

\author{
Correspondence \\ Claudio Cermelli \\ claudio.cermelli@unimore.it
}

Received 12 March 2009

Accepted 27 June 2009

\author{
Claudio Cermelli, ${ }^{1}$ Carlotta Francesca Orsi, ${ }^{1}$ Alessandro Cuoghi, \\ Andrea Ardizzoni, ${ }^{1}$ Enrico Tagliafico, ${ }^{2}$ Rachele Neglia, ${ }^{1}$ \\ Samuele Peppoloni ${ }^{1}$ and Elisabetta Blasi ${ }^{1}$
}
${ }^{1}$ Department of Public Health Sciences, University of Modena and Reggio Emilia, Via Campi 287, Modena, Italy
${ }^{2}$ Department of Biomedical Sciences, University of Modena and Reggio Emilia, Via Campi 287, Modena, Italy

\begin{abstract}
Recently, we showed that herpes simplex virus 1 (HSV-1)-infected monocytes have altered antifungal defences, in particular they show augmented phagocytosis of Candida albicans followed by a failure of the intracellular killing of the ingested fungi. On the basis of these functional data, comparative studies were carried out on the gene expression profile of cells infected with HSV-1 and/or C. albicans in order to investigate the molecular mechanisms underlying such virus-induced dysfunction. Affymetrix GeneChip technology was used to evaluate the cell transcription pattern, focusing on genes involved in phagocytosis, fungal adhesion, antimicrobial activity and apoptosis. The results indicated there was: (a) prevalent inhibition of opsonin-mediated phagocytosis, (b) upregulation of several pathways of antibody- and complement-independent phagocytosis, (c) inhibition of macrophage activation, (d) marked dysregulation of oxidative burst, (e) induction of apoptosis.
\end{abstract}

\section{INTRODUCTION}

Despite the increasing number of clinical reports on polymicrobial infections, little is known about the mutual interactions likely occurring among co-infecting pathogens and about their synergistic or antagonistic effects. Pathogen-to-pathogen interactions take place, directly and/or indirectly, and in turn, whenever they disturb host cell functions they may exacerbate the outcome of the infectious diseases. In many cases, polymicrobial infections involve herpesviruses (cytomegalovirus, Epstein-Barr virus, human herpesvirus 6 (HHV-6), herpes simplex virus 1 (HSV-1), Varicella-zoster virus) and fungi (Candida albicans, Cryptococcus neoformans, Pneumocystis carinii) (Karino et al., 1998; Rogers et al., 2000; Fonseca et al., 2003; Kobayashi et al., 2003; Marti Cabané \& Alvarez Rubio, 2004; Vuorinen et al., 2004; Weinberg et al., 2005). All herpesviruses are known to affect the immune system. In particular, HSV-1 can evade the host innate response through different mechanisms, including: (a) binding of complement components and immunoglobulin by envelope glycoproteins; (b) blocking of antigen presentation in

Abbreviations: FTL, ferritin L; HHV-6, human herpesvirus 6; HSV-1, herpes simplex virus 1; IFA, immunofluorescence assay; IFN, interferon; $\mathrm{NF} \kappa \mathrm{B}$, nuclear factor $\kappa \mathrm{B}$; ROS, reactive oxygen species; TLR, Toll-like receptor. the context of MHC molecules by expression of the viral ICP47; (c) inhibition of class I interferon (IFN) production; (d) impairment of dendritic cell maturation (Wakimoto et al., 2003).

Because of their role as the first-line defence against fungi, any dysfunction of macrophages is likely to be crucial in the evolution of mycoses. By an in vitro model, we have previously shown that in the course of mixed infections by viruses and fungi, viral infection of macrophages can alter their antifungal response. In particular, the THP-1 monocytic cell line exposed in vitro to HHV-6 (Cermelli et al., 2006) or HSV-1 (Cermelli et al., 2008), or to a transmissible cytotoxic activity (Beretti et al., 2007), shows dysregulation of antifungal defences. In all cases, phagocytosis of either Cryptococcus neoformans or C. albicans is significantly increased, but killing of the ingested fungi is markedly impaired, as proven by fungal survival and replication inside the macrophages. Moreover, alterations in the pattern of surface markers and secretory response are observed in the HSV-1-infected monocytic cells (Cermelli et al., 2008). In particular, Toll-like receptor (TLR)-2 and TLR-4, key molecules in fungal recognition, are downregulated; $\mathrm{CD} 38$ and $\mathrm{CD} 69$, well known markers of macrophage activation, and IL-12 production, critical in antifungal defences, are impaired; furthermore, markers of 
apoptosis and necrosis are increased. If such in vitro data mirror in vivo situations in which phagocytes, due to whatever factor, exhibit increased microbial ingestion followed by inefficient killing, we may speculate that such phenomenon will favour pathogen survival and dissemination, and ultimately disease progression. In fact, an unintended role of phagocytes as a 'Trojan horse' reservoir for the survival and dissemination of fungal cells has been proposed (Feldmesser et al., 2000).

In an attempt to unravel the molecular mechanisms leading to such herpesvirus-induced monocyte dysfunctions (Cermelli et al., 2006, 2008), we carried out comparative analyses on gene expression profiles of cells infected by HSV-1 and/or exposed to C. albicans. Affymetrix GeneChip technology was employed to focus on genes coding for molecules involved in phagocytosis, fungal adhesion, antimicrobial activity and apoptosis. A strong and wide-spectrum impact of HSV-1 infection on monocyte transcriptional pattern was observed. The parallelism between the present gene expression data and the previous phenotypical/functional findings (Cermelli et al., 2006, 2008) is detailed and the possible implications are discussed.

\section{METHODS}

Cell lines. The human acute monocytic leukaemia cell line THP-1 was maintained in RPMI 1640 medium with $10 \%$ fetal bovine serum, $2 \mathrm{mM}$ L-glutamine, $100 \mathrm{U}$ penicillin $\mathrm{ml}^{-1}$ and $100 \mu \mathrm{g}$ streptomycin $\mathrm{ml}^{-1}$, at $37{ }^{\circ} \mathrm{C}$ and $5 \% \mathrm{CO}_{2}$. Cells were split twice a week in fresh medium. The simian epithelial cell line Vero, used to propagate and titrate HSV-1, was cultivated in Eagle's minimal essential medium with the same components added as described above for RPMI 1640.

HSV-1. A clinical isolate obtained from blisters of a herpes labialis case, initially identified by indirect immunofluorescence assay (IFA) using a specific $\mathrm{mAb}$, was laboratory adapted by serial passages $(>50)$ through Vero cells. Viral stocks from THP-1 cells were prepared and harvested when most of the cells $(>80 \%)$ showed a cytopathic effect; the cell-free supernatants from the infected cultures were obtained by centrifugation and aliquots were stored at $-80{ }^{\circ} \mathrm{C}$. The virus stock titre was determined as p.f.u. $\mathrm{ml}^{-1}$ by the plaque method on Vero cells. Stocks for mock infection were prepared in the same manner from the supernatants of uninfected THP-1 cells.

C. albicans. The agerminative low-virulence strain PCa2 of $C$. albicans, a kind gift from Dr Kerridge (Department of Biochemistry, University of Cambridge, Cambridge, UK), was maintained by weekly passages on Sabouraud dextrose agar plates. Twenty-four hours before each experiment, C. albicans was transferred to a fresh plate; the following day, a few colonies were picked and resuspended in PBS for cell counting.

Infection with HSV-1 and Candida. THP-1 cells grown for $24 \mathrm{~h}$ were washed in PBS and then exposed to a suspension of HSV-1 at a m.o.i. of 10 p.f.u. per cell for $1 \mathrm{~h}$ at $37{ }^{\circ} \mathrm{C}$. After infection, THP- 1 cells

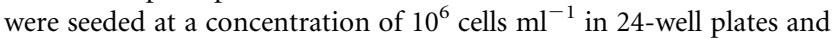
then incubated overnight. The following day, HSV-1-infected and -uninfected cultures were exposed to C. albicans $\left(10^{7}\right.$ cell ml ${ }^{-1}$, effector: target $=1: 10$ ) for $2 \mathrm{~h}$. Cultures were then centrifuged, washed once with PBS and frozen at $-70{ }^{\circ} \mathrm{C}$. Four experimental groups were prepared: mock-infected cells (group A), HSV-1-infected cells (group B), mock-infected cells exposed to C. albicans (group C) and HSV-1-infected cells exposed to C. albicans (group D). Each group was a pool of five different experiments giving a total of $5 \times 10^{6}$ cells per group. In parallel, a sample from each culture was used to determine the rate of HSV-1 infection of cells using IFA, according to a protocol reported elsewhere (Cermelli et al., 1992), by means of a $\mathrm{mAb}$ against HSV-1 gC glycoprotein (Argene).

RNA extraction and microarray hybridization. Total cellular RNA was isolated from THP-1 cells using an RNeasy RNA isolation kit (Qiagen) according to the manufacturer's instructions. Disposable RNA chips (Agilent RNA 6000 Nano LabChip kit) were used to determine the concentration and purity/integrity of RNA samples using an Agilent 2100 bioanalyser. cDNA synthesis, biotin-labelled target synthesis, and HG-U133 plus 2.0 GeneChip (Affymetrix) arrays hybridization, staining and scanning, were performed according to the standard protocol supplied by Affymetrix, and using the one cycle cDNA synthesis kit. The amount of a transcript mRNA (signal) was determined by the GCOS (GeneChip operating software) absolute analysis algorithm as described by Liu et al. (2002). All expression values for the genes in the GCOS absolute analyses were determined using the global scaling option. The Affymetrix GCOS comparison algorithm was used for supervised analysis (Liu et al., 2002). Microsoft Excel was then used for filtering modulated transcripts.

Gene ontology analysis. Gene ontology analysis was performed using Ingenuity Pathways Analysis software (Ingenuity Systems, www.ingenuity.com). In particular, we identified the biological functions that were most significant among the modulated gene dataset, considering genes that met the cut-off of $>2$ or $<-2(\log 2$ ratio) and were associated with a canonical pathway in the Ingenuity Pathways Knowledge Base. The significance of the association between the dataset and the canonical pathway was measured as the ratio between the number of genes from the dataset that map to the pathway and the total number of genes that map to the canonical pathway. Fischer's exact test was used to calculate the $P$ value.

\section{RESULTS}

\section{Infection of THP-1 cells with HSV-1}

Preliminary IFA experiments were carried out in order to evaluate the susceptibility of THP-1 cells to HSV-1 infection. In particular, the rate of HSV-1 infection of THP-1 cells was assessed after exposure to cell-free viral stocks testing three m.o.i. and different time points. Based upon the results obtained (data not shown), we chose the m.o.i. of 10 p.f.u. per cell and $18 \mathrm{~h}$ incubation, since under these conditions more than $80 \%$ of THP-1 cells were positive for a HSV-1 late antigen (gC).

\section{Gene expression profiling of infected THP-1 cells}

Gene expression profiling was carried out using Affymetrix GeneChip technology as described in Methods. In particular, four groups of THP-1 cells were assessed: group A consisted of mock-infected cells (negative controls) incubated for $18 \mathrm{~h}$; group B consisted of cells exposed to HSV-1 for $18 \mathrm{~h}$; in group C cells were exposed to mockinocula for $16 \mathrm{~h}$ and then to C. albicans for $2 \mathrm{~h}$; in group $\mathrm{D}$, cells were exposed to HSV-1-inocula for $16 \mathrm{~h}$ and then to $C$. albicans for $2 \mathrm{~h}$. A preliminary rough analysis 
indicated a remarkable shut off of THP-1 gene expression by HSV-1, since in HSV-1-infected cells (groups B and D) only $19 \%$ of the 54876 probe sets studied were present, while in cells not exposed to HSV-1 (groups A and C) the number of probe sets present was more than double (>40\%) (Fig. 1). The impact of the single or dual infection on cell transcription is detailed in Fig. 2. HSV-1 infection caused a marked change in THP-1 gene expression, with about 3000 probe sets upregulated and more than 4600 downregulated in comparison with uninfected THP-1 cells (group B vs group A). The exposure of THP-1 cells to $C$. albicans for $2 \mathrm{~h}$ (group $\mathrm{C}$ vs group $\mathrm{A}$ ) caused expression changes only in a limited number of genes (approx. 400 upregulated and 100 downregulated). The comparison between cells with dual infection and the negative controls (group D vs group A) was similar to that between HSV-1infected cells and negative controls (approx. 3000 upregulated and 4800 downregulated).

The functional mapping of modulated genes was carried out using Ingenuity Pathways Analysis software. The results of analyses obtained (data not shown) suggested a modulation of the functional categories of genes related to the control of cell proliferation and/or cell death. A more in-depth analysis was performed focusing on a selected number of gene clusters related to monocyte-mediated effector functions.

Fig. 3 shows details of the gene expression changes observed in cells with single HSV-1 infection versus negative control cells (group B vs group A); data are clustered in five classes according to the role of each gene product in monocyte functions (opsonized phagocytosis, antibody- and complement-independent phagocytosis, antimicrobial activity, oxidative burst, apoptosis). In

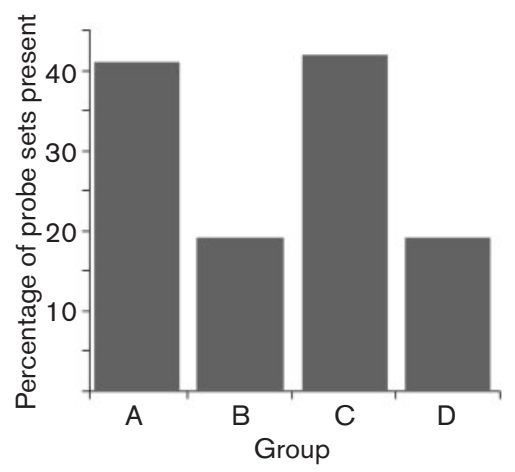

Fig. 1. Percentage of probe sets present in the different experimental cell groups. Groups: A, uninfected cells incubated for 18 h; B, THP-1 cells infected with HSV-1 at a m.o.i. of 10 p.f.u. per cell for $18 \mathrm{~h}$; C, THP-1 cells incubated for $16 \mathrm{~h}$ and then infected with $C$. albicans $\left(10^{7}\right.$ cell $\mathrm{ml}^{-1}$, effector : target $\left.=1: 10\right)$ for $2 \mathrm{~h}$; D, THP-1 cells infected with HSV-1 at a m.o.i. of 10 p.f.u. per cell for $16 \mathrm{~h}$ and then infected with C. albicans for $2 \mathrm{~h}$. After $18 \mathrm{~h}$ incubation in 24-well plate, total RNA was extracted and gene expression analysed by Affimetrix GeneChip technology.

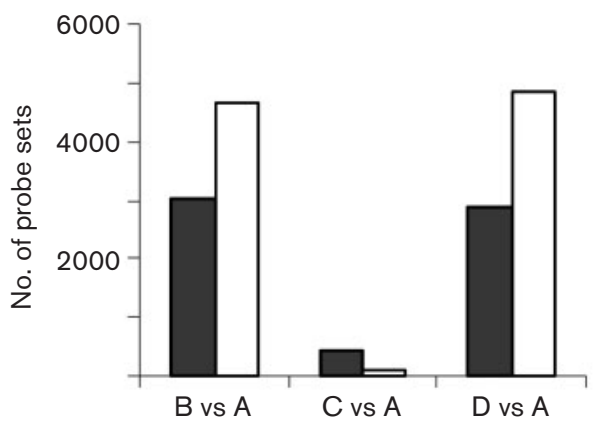

Fig. 2. Impact of single or dual infection on THP-1 gene expression. A, Uninfected cells incubated for $18 \mathrm{~h}$; B, THP-1 cells infected with HSV-1 at a m.o.i. of 10 p.f.u. per cell for $18 \mathrm{~h}$; C, THP-1 cells incubated for $16 \mathrm{~h}$ and then infected with $C$. albicans $\left(10^{7}\right.$ cell $\mathrm{ml}^{-1}$, effector : target $\left.=1: 10\right)$ for $2 \mathrm{~h}$; D, THP-1 cells infected with HSV-1 at a m.o.i. of 10 p.f.u. per cell for $16 \mathrm{~h}$ and then infected with C. albicans for $2 \mathrm{~h}$. After $18 \mathrm{~h}$ incubation in 24well plate, total RNA was extracted and gene expression analysed by Affimetrix GeneChip technology. Grey bars, probe sets with increased expression; white bars, probe sets with decreased expression.

particular, HSV-1 infection strongly affected the expression of certain genes involved in phagocytosis. The first class comprised genes related to immunoglobulin and complement-mediated phagocytosis, which were prevalently either unchanged or downregulated in HSV-1infected THP-1 cells. This class includes genes involved in fungal adhesion and up-take, such as integrins and other complement receptors (CD18, CD29, CD44, CD49, CD103), Fcy receptors (CD32, CD64), lectins (CD33, mannose binding molecules), RHO GTPase proteins (CDC42 proteins and RAC-1, which are downstream mediators of $\mathrm{F} c \gamma$ receptors). The second class comprised genes involved in antibody-independent phagocytosis, which were predominantly upregulated, namely, important molecules in candida recognition, such as MASP2, some genes of the tyrosine kinase pathways (FYN, CAV1), proteins involved in cytoskeleton modifications (ACTN4) and vesicle trafficking (BMX, RUFY1), and CLEC4A, a Ctype lectin. In the third class a wide-spectrum downregulation was observed among genes involved in killing and antimicrobial activity, including many genes linked to: (a) endosome maturation and phago-lysosome fusion (LAMP1 and LAMP2, CD63, RAB9a, RAB7); (b) the nuclear factor $\kappa \mathrm{B}(\mathrm{NF} \kappa \mathrm{B})$ signalling pathway from TLR-4 (BCL-10, IRAK-1, CARD9, SYK); (c) various signalling pathways for cell activation (MAPK1, CD38); (d) transporters (SLC7A1, SLC7A7, SLC37A4, ABCC1); (e) chemokines and chemokine receptors (CX3CR, CCL5/RANTES). The fourth group of genes included those related to the oxidative burst. Two key genes involved in $\mathrm{H}_{2} \mathrm{O}_{2}$ production, NOX3 and NOX4, were upregulated in HSV-1-infected monocytic cells, whereas many enzymes responsible for the neutralization of reactive oxygen species 
Fold change (log ratio)

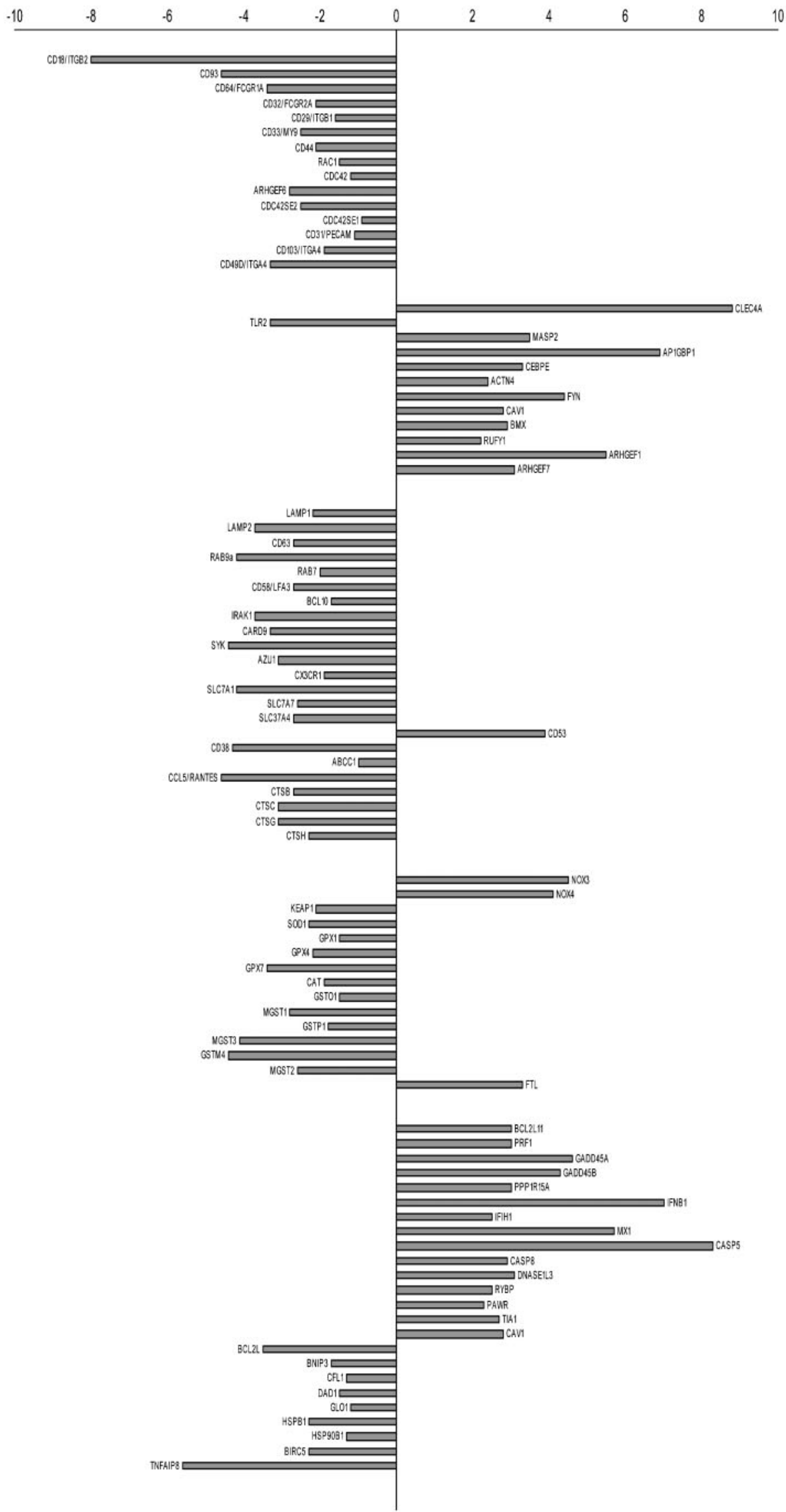


(ROS) were downregulated. In particular, we found a downregulated expression of peroxidases, catalases, superoxide dismutases, glutathione transferases, as well as the KEAP-1 gene, a promoter of genes known to be involved in cell protection from oxidative stress. However, ferritin L (FTL) was overexpressed. Finally, the fifth class comprised more than 200 genes among those related to apoptotic pathways, which were strongly affected by HSV-1 infection (the most significant ones are shown in Fig. 3). In particular, several apoptosis inducers and positive regulators were upregulated: among these BCL2L11, GADD45A, GADD45B, IFN- $\beta 1$, MX1, CASP5, CASP8. On the contrary, antiapoptotic genes and negative regulators were downregulated, namely BCL2, HSPB1, HSP90B1, TNFAIP8 and BIRC5.

Fig. 4 shows the genes, among those considered in the analysis shown in Fig. 3, whose expression was modulated in THP-1 cells upon exposure to C. albicans, in comparison with uninfected controls (group C vs group A). There were only five such genes.

Finally, we directly compared THP-1 cells infected by HSV-1 plus Candida with those infected by HSV-1 alone (group D vs group B). As shown in Fig. 5, for most of them, little $(<1 \log )$ or no variation was observed, irrespective of the clusters of genes considered.

\section{DISCUSSION}

As previously described (Cermelli et al., 2006, 2008), THP1 monocytic cells infected either with HHV-6 or HSV-1 displayed altered antifungal defences; in both cases, augmented phagocytosis of the yeast cells is followed by a failure in the intracellular killing of the ingested fungi; as a consequence, an increase in survival and replication of the pathogens occurs (Cermelli et al., 2006, 2008). Employing the same in vitro infection model, herein we describe the gene expression profile of a monocytic cell line infected with HSV-1 and subsequently exposed or not exposed to $C$. albicans. As shown in Fig. 2, the virus per se had a major impact on monocyte gene expression: about 3000 probe sets were upregulated and more than 4600 were downregulated in HSV-1-infected cells with respect to the control cells. In line with this result, the percentage of expressed genes in the cell populations was different, namely, $19 \%$ in the infected cells versus $41 \%$ in the controls. In contrast, in the cells infected with C. albicans alone, the expression of only few hundred genes is altered, namely approximately 400 are upregulated and 100 are downregulated, and the percentage of expressed genes
( $42 \%)$ is similar to the one found in the control cells. The difference observed between virus- and fungal-infected cells is not surprising due to (a) the different infection times (18 h for HSV-1 vs $2 \mathrm{~h}$ for Candida), which have been chosen according to the previously described phenotypical studies (Cermelli et al., 2008), and (b) the intimate relation occurring between virus and host cell. In this respect, HSV-1 causes a shut off of many cell functions through its virion-host shut off protein, an RNase coded by UL41, which heavily affects cellular RNAs degrading preexisting and newly transcribed mRNAs (Smiley, 2004; Poon et al., 2006). In any case, while kinetics studies are needed to elucidate the relevance of infection time per se, the present data indicated that, at a given time point, namely when THP-1 cells are dysregulated because of the virus infection (Cermelli et al., 2008), thousands of genes are affected; furthermore, they remain approximately as such irrespectively of the fact that monocytes have to deal with a superinfecting agent (Figs 1 and 4).

Gene ontology analysis revealed that most of the modulated genes were not surprisingly related to cell proliferation and/ or cell death. More informative datasets were obtained focusing on a selected pool of genes. In particular, the expression analysis of genes involved in phagocytosis revealed interesting results. Firstly, many genes playing a crucial role in opsonin-dependent phagocytosis were downregulated by HSV-1 (Fig. 3). Several of them encode molecules involved in fungal adhesion, such as $\beta$-integrins and receptors for the $\mathrm{Fc}$ fragment and for complement components. In particular, CD93, a crucial modulator of the phagocytosis of opsonized fungi, as well as many components of pathways involved in the $\mathrm{F} c \gamma$ receptor-mediated phagocytosis (CD64, CD18, CD29, CD49, CD103), are downregulated. Another important pathway of opsoninmediated phagocytosis involved CD44. CD44 is a receptor that efficiently mediates internalization of large particles involving Syk and Rac1, which in turn lead to MAPK activation via CDC42 (Vachon et al., 2006). Also these gene transcripts are decreased in HSV-1-infected monocytes. On the whole, we may speculate that such a downregulation is a monocyte strategy to avoid meaningless reactions, since the infection model employed in this study is devoid of opsonizing factors, all the machinery related to opsonindependent phagocytosis, not being required, is properly shut-off or not activated.

Secondly, the expression of many genes involved in antibody- and complement-independent phagocytosis is markedly modulated by HSV-1. In particular, TLR-2 gene expression is downregulated; this finding fully matches our previous functional data revealing that HSV-1-infected

Fig. 3. Comparison of the gene expression of selected genes in HSV-1-infected-cells compared to -uninfected cells. THP-1 cells were infected or not infected with HSV-1 at a m.o.i. of 10 p.f.u. per cell for $18 \mathrm{~h}$ in 24-well plate. Then, total RNA was extracted and gene expression analysed by Affimetrix GeneChip technology. The expression changes of selected genes related to five different classes of macrophage functions are shown; the changes are expressed as the log ratio between the signal of HSV-1-infected cells and that of uninfected controls. 


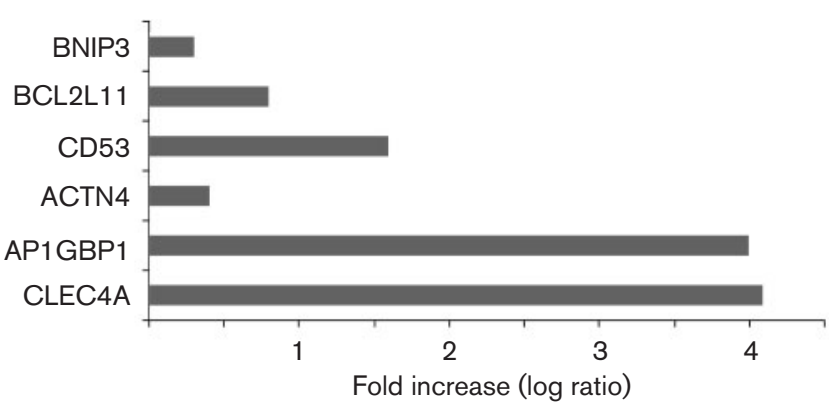

Fig. 4. Comparison of the expression of selected genes in $C$. albicans-infected cells compared to uninfected cells. THP-1 cells were incubated for $16 \mathrm{~h}$ in 24-well plate and then infected or not infected with C. albicans $\left(10^{7}\right.$ cell $\mathrm{ml}^{-1}$, effector : target $\left.=1: 10\right)$ for $2 \mathrm{~h}$. Then, total RNA was extracted and gene expression analysed by Affimetrix GeneChip technology. In Candida-infected cells, only five genes among those shown in Fig. 2 displayed an expression change (expressed as log ratio) in comparison with uninfected controls.

macrophages have a lower percentage of TLR-2 positive cells and reduced mean fluorescent intensity with respect to uninfected controls, as assessed by flow cytometry analysis (Cermelli et al., 2008). Such a decrease in TLR-2 is accompanied by an increased phagocytosis, implying an inverse relation between TLR-2 expression and phagocytic activity. Accordingly, by means of another functional study, we have shown that TLR-2 knockout macrophages exhibit a significantly lower ability in ingesting fungi with respect to a TLR-2 $+/+$ counterpart (Blasi et al., 2005). Thus, among the plethora of controversial data on TLR-2, our present transcriptional results closely fit with the functional data previously published (Cermelli et al., 2006, 2008) and unanimously strengthen the hypothesis that TLR-2 is somehow detrimental in Candida recognition/ phagocytosis. Furthermore, TLR-2 recognition/signalling is a known mediator of the HSV-1-induced immune response leading to inflammatory burst and, subsequently, to apoptosis (Mansur et al., 2005). Likely, the TLR-2 downregulation observed here after virus infection is one of the mechanisms used by HSV-1 to contain the monocyte reaction, and may contribute to the observed failure in the cell activation and killing of the ingested fungi. In the present study, we showed that MASP2 gene expression is upregulated upon virus infection. MASP2 is a protease somehow involved in phagocytosis, since it binds $C$. albicans via a serum mannose-binding lectin (Held et al., 2008). Many genes whose products are involved in engulfment, endosome formation, vesicle trafficking and cytoskeletal rearrangements (such as AP1GBP1, ACTN4, FYN, CAV1, ARHGEF1, ARHGEF7 BMX and RUFY1) are also overexpressed in HSV-1-infected monocytes. Such a wide-spectrum impact of HSV-1 on the infected cells can be explained by its need to mobilize viral components within the host cell, especially at the membrane level. Finally, we showed that CLEC4A gene expression is highly upregulated in monocytes infected with either HSV-1 (Fig. 3 ) or C. albicans alone (Fig. 4). Once more, these transcriptional findings fit with the observed increase in Candida uptake (Cermelli et al., 2008). Since CLEC4A is a poorly characterized C-type lectin receptor recognizing carbohydrate moieties on pathogens (Areschoug \& Gordon, 2008; Meyer-Wentrup et al., 2008), we speculate that such a pathogen recognition receptor plays a role in $C$. albicans recognition and uptake.

Based on functional studies demonstrating impaired killing and increased apoptosis by HSV-1 (Cermelli et al., 2006, 2008) we focused on the expression of genes related to these pathways. With respect to antifungal activity, all but one of the genes endowed with endosome maturation/ acidification (LAMP1, LAMP2, CD63, RAB9a, RAB7), as well as with antimicrobial activity (azurocidin 1 and cathepsins), were markedly reduced in the virus-infected cell. Also genes encoding important surface molecules, such as CD38 and CD58, were downregulated. CD38 is a multifunctional enzyme involved in monocyte activation and proliferation (De Flora et al., 2004; Malavasi et al., 2006). The transcriptional inhibition of CD38 observed here expands our previous report showing a reduction of this protein at the membrane level, as assessed by flow cytometry analysis (Cermelli et al., 2008). Finally, HSV-1 markedly downregulated BCL10 and CARD9, whose products are involved in immune cell activation via $\mathrm{NF} \kappa \mathrm{B}$. It is noteworthy that it has been shown that CARD9 knockout mice are significantly more susceptible to C. albicans infection than wild-type mice, and CARD9deficient dendritic cells display defective activation and an impaired cytokine response to zymosan stimulation (Gross et al., 2006). Thus, taken together our transcriptional data, revealing downregulation of $\mathrm{NF} \kappa \mathrm{B}$ and CD38 in monocytes infected by HSV-1 may explain functional results showing a virus-induced impairment of antifungal capabilities (Cermelli et al., 2006, 2008).

Unlike the other genes involved in antimicrobial activity, CD53 is upregulated in HSV-1-infected cells. CD53 gene expression is known to be enhanced in LPS-treated monocytes; interestingly, such cells are in turn protected from oxidative stress due to CD53 counteraction of LPSinduced ROS inside the cells (Kim et al., 2004). We speculate that the CD53 overexpression, observed also under our experimental conditions, contributes to $C$. albicans survival inside monocytes via diminution of ROS. Similarly, FTL transcription is increased in our infection model; since FTL is reported to reduce ROS in response to oxidant challenge (Orino et al., 2001), we speculate that its upregulation, combined with that of CD53, may account for both $\mathrm{H}_{2} \mathrm{O}_{2}$ and ROS neutralization. Conversely, the upregulation of NOX3 and NOX4 genes suggests a scenario of increased $\mathrm{H}_{2} \mathrm{O}_{2}$ production in a context of general inhibition of the mechanisms leading to neutralization of ROS generated from $\mathrm{H}_{2} \mathrm{O}_{2}$ (decrease in KEAP-1, peroxidases, catalases, superoxide dismutases, glutathione transferases). The net result of such conflicting patterns, 


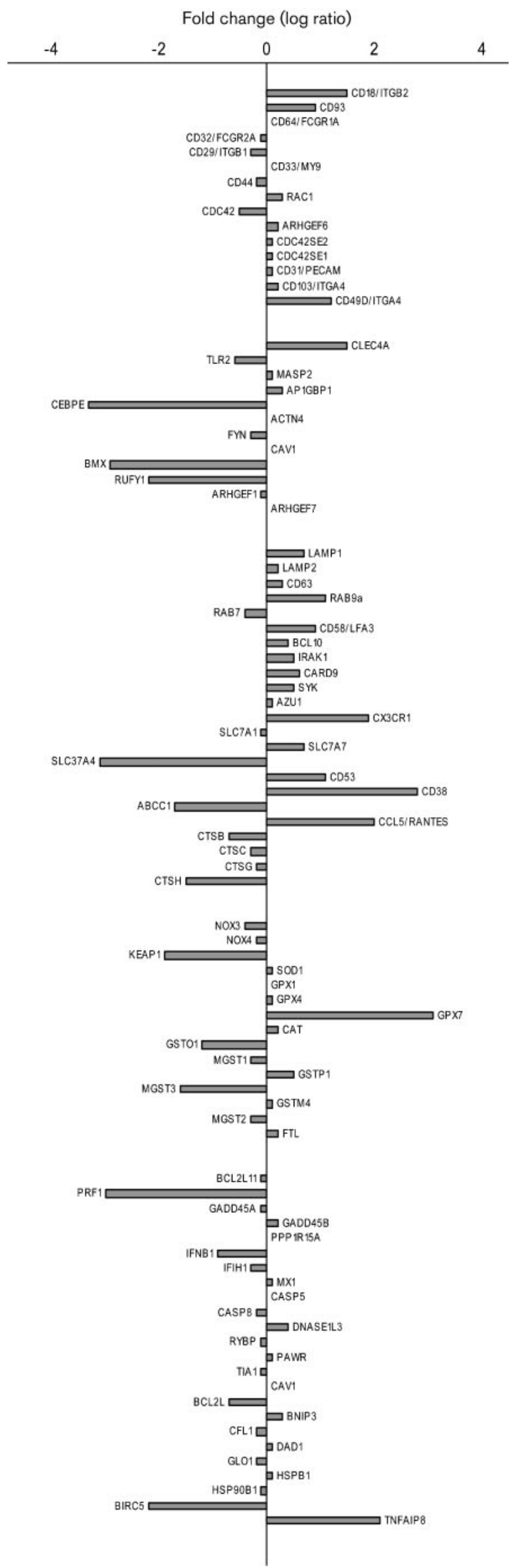

Fig. 5. Comparison of the gene expression of selected genes in cells with dual (HSV-1 + Candida) and single (HSV-1) infection. THP-1 cells were infected with HSV-1 at a m.o.i. of 10 p.f.u. per cell for $16 \mathrm{~h}$ in 24-well plate; afterwards, they were exposed or not exposed to C. albicans $\left(10^{7}\right.$ cell $\mathrm{ml}^{-1}$, effector : target $\left.=1: 10\right)$ for an additional $2 \mathrm{~h}$. Then total RNA was extracted and gene expression analysed by Affimetrix GeneChip technology. In this gene expression comparison, only the genes among those shown in Fig. 2 displaying an expression change (expressed as log ratio) between the two groups are reported.

observed at the transcriptional level, may be deduced on the bases of the functional data (Cermelli et al., 2006, 2008): in the balance between production of antimicrobial molecules (ROS) and self protection from oxidative burst occurring upon virus infection, the secondary expression patterns, including CD53 and FTL, prevail becoming beneficial, in turn, to the ingested fungus.

CCL5/RANTES has been shown to directly exert both anticandida and anti-HSV-1 activity (Tang et al., 2002; Nakayama et al., 2006); therefore, it is not surprising that it is downregulated in our model of HSV-1-infected THP-1 cells, previously established as fully permissive (Cermelli et al., 2008). Such a phenomenon may be considered as another pathogen-induced strategy to bypass monocyte reactions. Accordingly, by another model employing human microglia, Marques et al. (2008) showed that, when RANTES is induced, HSV-1 replication is fully inhibited.

The failure in anti-candida capabilities observed in HSV-1infected THP-1 cells can also be explained considering the pattern of gene expression related to apoptosis. A plethora of apoptosis inducers and positive regulators are increased, whereas antiapoptotic genes and negative regulators are decreased, upon infection. Thus, these transcriptional results, which are in line with our previous phenotypebased report showing an increase of apoptotic markers in HSV-1-infected THP-1 cells (Cermelli et al., 2008), further suggest a direct role for apoptosis in the impaired monocyte activation and antimicrobial functions.

Finally, it must be considered that virus-induced cell products may, in turn, alter cellular gene expression. This may be particularly true considering the long-term infection employed in the present work. For example, HSV-1 induces IFN production, which then strongly affects cellular gene expression. In THP-1 cells, we observed augmented IFN $\beta 1$ and IFN $\gamma$ receptor (IFNGR) mRNAs. Interestingly, a higher expression of IFNGR can contribute to the potentiation of phagocytosis.

In conclusion, using microarray technology, a widespectrum analysis has been performed. Although we are aware of the limitation of such an approach, the parallelism observed between the transcription profiling and the phenotypical/functional data strongly supports the overall 
picture of a monocyte profoundly affected by HSV-1 in its response(s) when it is superinfected by a pathogen that shares the monocyte as a target cell.

\section{ACKNOWLEDGEMENTS}

This work was supported by a grant from MIUR (Ministero dell' Istruzione, dell' Università e della Ricerca Scientifica) [PRIN (Progetto di Rilevante Interesse Nazionale) 2007 no. 20072CF9L2002].

\section{REFERENCES}

Areschoug, T. \& Gordon, S. (2008). Pattern recognition receptors and their role in innate immunity: focus on microbial protein ligands. Contrib Microbiol 15, 45-60.

Beretti, F., Cenacchi, V., Portolani, M., Ardizzoni, A., Blasi, E. \& Cermelli, C. (2007). A transmissible cytotoxic activity isolated from a patient with brain ischemia causes microglial cell activation and dysfunction. Cell Mol Neurobiol 27, 517-528.

Blasi, E., Mucci, A., Neglia, R., Pezzini, F., Colombari, B., Radzioch, D., Cossarizza, A., Lugli, E., Volpini, G. \& other authors (2005). Biological importance of the two Toll-like receptors, TLR2 and TLR4, in macrophage response to infection with Candida albicans. FEMS Immunol Med Microbiol 44, 69-79.

Cermelli, C., Moroni, A., Pietrosemoli, P., Pecorari, M. \& Portolani, M. (1992). IgG antibodies to human herpesvirus-6 (HHV-6) in Italian people. Microbiologica 15, 57-63.

Cermelli, C., Cenacchi, V., Beretti, F., Pezzini, F., Luca, D. D. \& Blasi, E. (2006). Human herpesvirus-6 dysregulates monocyte-mediated anticryptococcal defences. J Med Microbiol 55, 695-702.

Cermelli, C., Orsi, C. F., Ardizzoni, A., Lugli, E., Cenacchi, V., Cossarizza, A. \& Blasi, E. (2008). Herpes simplex virus type 1 dysregulates anti-fungal defenses preventing monocyte activation and downregulating Toll-like receptor-2. Microbiol Immunol 52, 575-584.

De Flora, A., Zocchi, E., Guida, L., Franco, L. \& Buzzone, S. (2004). Autocrine and paracrine calcium signaling by the CD38/NAD ${ }^{+} /$cyclic ADP-ribose system. Ann N Y Acad Sci 1028, 176-191.

Feldmesser, M., Kress, Y., Novikoff, P. \& Casadevall, A. (2000). Cryptococcus neoformans is a facultative intracellular pathogen in murine pulmonary infection. Infect Immun 68, 4225-4237.

Fonseca, E. M., Nuno, F. J., Garcia-Alcalde, M. L. \& Menendez, M. J. (2003). Infection due to herpes zoster and cryptococcus after initiating high-activity antiretroviral treatment. Enferm Infecc Microbiol Clin 21, 217-218 (in Spanish).

Gross, O., Gewies, A., Finger, K., Schafer, M., Sparwasser, T., Peschel, C., Forster, I. \& Ruland, J. (2006). Card9 controls a non-TLR signalling pathway for innate anti-fungal immunity. Nature 442, 651656.

Held, K., Thiel, S., Loos, M. \& Petry, F. (2008). Increased susceptibility of complement factor $\mathrm{B} / \mathrm{C} 2$ double knockout mice and mannanbinding lectin knockout mice to systemic infection with Candida albicans. Mol Immunol 45, 3934-3941.

Karino, T., Nakamura, J., Fujita, K., Kobashi, Y., Yano, T., Okimoto, N. \& Soejima, R. (1998). A case with chronic active EB virus infection accompanied with pulmonary candidiasis. Kansenshogaku Zasshi 72, 1306-1310 (in Japanese).

Kim, T. R., Yoon, J. H., Kim, Y. C., Yook, Y. H., Kim, I. G., Kim, Y. S., Lee, H. \& Paik, S. G. (2004). LPS-induced CD53 expression: a protection mechanism against oxidative and radiation stress. Mol Cells 17, 125-131.

Kobayashi, T. K., Ueda, M., Nishino, T., Moritani, S., Higaki, T. \& Bamba, M. (2003). Cytologic detection of cryptococcosis coexisting with herpes simplex virus infection in sputum: use of liquid-based, thin-layer preparations. Acta Cytol 47, 103-106.

Liu, W. M., Mei, R., Di, X., Ryder, T. B., Hubbell, E., Dee, S., Webster, T. A., Harrington, C. A., Ho, M. H. \& other authors (2002). Analysis of high density expression microarrays with signed-rank call algorithms. Bioinformatics 18, 1593-1599.

Malavasi, F., Deaglio, S., Ferrero, E., Funaro, A., Sancho, J., Ausiello, C. M., Ortolan, E., Vaisitti, T., Zubiaur, M. \& other authors (2006). CD38 and CD157 as receptors of the immune system: a bridge between innate and adaptive immunity. Mol Med 12, 334-341.

Mansur, D. S., Kroon, E. G., Nogueira, M. L., Arantes, R. M., Rodrigues, S. C., Akira, S., Gazzinelli, R. T. \& Campos, M. A. (2005). Lethal encephalitis in myeloid differentiation factor 88-deficient mice infected with herpes simplex virus 1. Am J Pathol 166, 1419-1426.

Marques, C. P., Cheeran, M. C., Palmquist, J. M., Hu, S., Urban, S. L. \& Lokensgard, J. R. (2008). Prolonged microglial cell activation and lymphocyte infiltration following experimental herpes encephalitis. J Immunol 181, 6417-6426.

Marti Cabané, J. \& Alvarez Rubio, M. (2004). Herpes simplex esophagitis associated to Candida albicans in an immunocompetent host. An Med Interna 21, 312 (in Spanish).

Meyer-Wentrup, F., Benitez-Ribas, D., Tacken, P. J., Punt, C. J., Figdor, C. G., de Vries, I. J. \& Adema, G. J. (2008). Targeting DCIR on human plasmacytoid dendritic cells results in antigen presentation and inhibits IFN- $\alpha$ production. Blood 111, 4245-4253.

Nakayama, T., Shirane, J., Hieshima, K., Shibano, M., Watanabe, M., Jin, Z., Nagakubo, D., Saito, T., Shimomura, Y. \& Yoshie, O. (2006). Novel antiviral activity of chemokines. Virology 350, 484-492.

Orino, K., Lehman, L., Tsuji, Y., Ayaki, H., Torti, S. V. \& Torti, F. M. (2001). Ferritin and the response to oxidative stress. Biochem J 357, 241-247.

Poon, A. P., Gu, H. \& Roizman, B. (2006). ICP0 and the US3 protein kinase of herpes simplex virus 1 independently block histone deacetylation to enable gene expression. Proc Natl Acad Sci U S A 103, 9993-9998.

Rogers, J., Rohal, S., Carrigan, D. R., Kusne, S., Knox, K. K., Gayowski, T., Wagener, M. M., Fung, J. J. \& Singh, N. (2000). Human herpesvirus- 6 in liver transplant recipients: role in pathogenesis of fungal infections, neurologic complications, and outcome. Transplantation 69, 2566-2573.

Smiley, J. R. (2004). Herpes simplex virus virion host shutoff protein: immune evasion mediated by a viral RNase? J Virol 78, 1063-1068.

Tang, Y. Q., Yeaman, M. R. \& Selsted, M. E. (2002). Antimicrobial peptides from human platelets. Infect Immun 70, 6524-6533.

Vachon, E., Martin, R., Plumb, J., Kwok, V., Vandivier, R. W., Glogauer, M., Kapus, A., Wang, X., Chow, C. W. \& other authors (2006). CD44 is a phagocytic receptor. Blood 107, 4149-4158.

Vuorinen, T., Kotilainen, P., Lautenschlager, I., Kujari, H., Krogerus, L. \& Oksi, J. (2004). Interstitial pneumonitis and coinfection of human herpesvirus 6 and Pneumocystis carinii in a patient with hypogammaglobulinemia. J Clin Microbiol 42, 5415-5418.

Wakimoto, H., Johnson, P. R., Knipe, D. M. \& Chiocca, E. A. (2003). Effects of innate immunity on herpes simplex virus and its ability to kill tumor cells. Gene Ther 10, 983-990.

Weinberg, A., Bloch, K. C., Li, S., Tang, Y. W., Palmer, M. \& Tyler, K. L. (2005). Dual infections of the central nervous system with EpsteinBarr virus. J Infect Dis 191, 234-237. 